\title{
Ashra Optical Transient Observation
}

\author{
H. Oshima* \\ Department of Physics, Toho University, Funabashi, Chiba 274-8510, Japan \\ E-mail: 74160010 enc.toho-u.ac.ip
}

Ashra (All-sky Survey High Resolution Air-shower detector) is a project to build an unconventional optical telescope complex that images very wide field of view, covering $77 \%$ of the sky. In regard to the plateau region of Gamma-ray burst (GRB) afterglow, various models for explanation exist, however its origin is still not sure. There is a model that can explain this region by the precursor component earlier than the start time of GRB. We analyzed observation data of Ashra-1 to study optical flash of precursor.

35th International Cosmic Ray Conference - ICRC2017

10-20 July, 2017

Bexco, Busan, Korea

${ }^{*}$ Speaker. 


\section{Introduction}

Gamma ray bursts are the largest explosion phenomenon in the universe, which emit gamma rays with enormous amounts of energy in a very short time. Currently, the widely accepted GRB standard theoretical model is a relativistic fireball model[四. According to the fireball model, a large amount of energy is generated from the engine which is an energy supply source. And a fireball consisted of photons, electrons, positrons, and baryons is generated. As the fireball expands, the internal energy is transformed into the kinetic energy of the baryon contained in the fireball. Spherical shell expanding at a relativistic velocity is formed. At first, light can't be emitted from a fireball because of its high density. But as the expansion of the shell breaks the surface of the star and moves to the outside, the density decreases. After decreasing density, light can be emitted from a fireball. We understand that GRB is caused by internal shock wave and afterglow is caused by external shock wave. Since there is a difference in velocity between the matter in the expansion, they collide with each other during the flight. At this time, an internal shock wave is emitted. This internal shock wave accelerates a particles and generates a magnetic field. And gamma rays are radiated nonthermally via synchrotron radiation[[]]. After that, when the ejected matter collides with surrounding interstellar matter, an external shock wave is generated. With this external shock wave, afterglows of X-ray and optical wavelength is emitted[B]].

Fluxes of X-ray afterglows can be divided into three regions depending on time. The first phase is a steeply decreasing region, the second phase is a flat plateau region, and the third phase is a normal decay region. According to the widely believed theory in general, the first region is

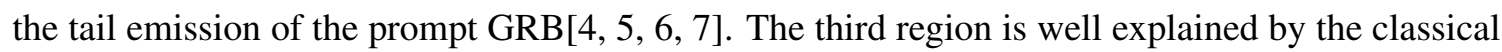
external shock model[ $[8]$. In respect of the second region, various models for explanation exist, however its origin is still not sure. There is a model that can explain this plateau region by the precursor component earlier than the start time of GRB $\left(\mathrm{t}_{0}\right)$ [Q] .

\section{Ashra Experiment and Detector}

Ashra (All-sky Survey High Resolution Air-shower detector) is a project to build an unconventional optical telescope complex that images very wide field of view, covering $77 \%$ of the sky. Ashra light collector has a $42^{\circ}$ diameter field of view and a few arcminutes by using the image intensifier[ए0, [1] and CMOS technology. The project primarily aims to observe cherenkov and fluorescence lights from the lateral and longitudinal developments of very high energy cosmic rays in the atmosphere. We reported the observational search for tau neutrinos $\left(v_{\tau}\right)$ from GRB081203A using one of the Ashra light collectors[[2]. The Earth-skimming $v_{\tau}$ technique of imaging cherenkov $\tau$ showers was applied as a detection method. However, our detectors can also be used to monitor optical transients in the wide field of sky. The demonstration phase, Ashra-1, has been running at the Mauna Loa site (3300m a.s.1.) in Hawaii since 2008. Ashra-1 had completed its 3rd observation period, the first dedicated to taking physics data for PeV-EeV tau neutrinos with the best instantaneous sensitivity and optical transients, in March 2013. From January 2012 until end of February 2013, about 1950 hours of data have been taken out of 2006 hours possible due to light constraints. For optical transients, we have 3763 additional hours of data taken from 2008 until 2011. 


\section{Search for Precursor Candidates}

We can analyze an optical flush of GRB by using the Ashra light collector having a wide field of view(FOV) and high resolution. In particular, by taking advantage of our detector, it is possible to analyze precursor. We analyzed observation data of Ashra-1 to study optical flash of precursor. The light collectors used in this analysis have the achieved resolution of a few arcmin, viewing $42^{\circ}$ circle region of which center are located at $($ Alt, Az $)=\left(11.7^{\circ}, 22.1^{\circ}\right),\left(60.0^{\circ}, 0.0^{\circ}\right)$. We extracted the analysis candidates of precursors based on alert information of Swift satellite[[ए3] and Fermi Gamma-ray Space Telescope[[4]]. The time window is $t_{0}-24$ hours to $t_{0}+1$ hour, here, $t_{0}$ is the time when GRB was triggered. We imposed the condition that angular distance from the center of FOV to GRB including the 3 sigma of angle error of each satellite is less than $21^{\circ}$. Finally, we extracted 59 precursor candidates (Swift : 40 candidates, Fermi : 19 candidates) with confidence level $99.7 \%$. The Figure $\square$ shows the our precursor candidates in equatorial coordinate system. The Figure $\square$ shows the candidate's trajectories in horizontal coordinate of our observation system. It can be seen that the GRB before $t_{0}$ is within the observation field of view. Analysis of the extracted precursor candidates is now in progress.



Figure 1: The precursor candidates in equatorial coordinate system. The magenta star marks represent the GRBs detected by Swift satellite and the cyan star marks represent the GRBs detected by Fermi Gamma-ray Space Telescope. 


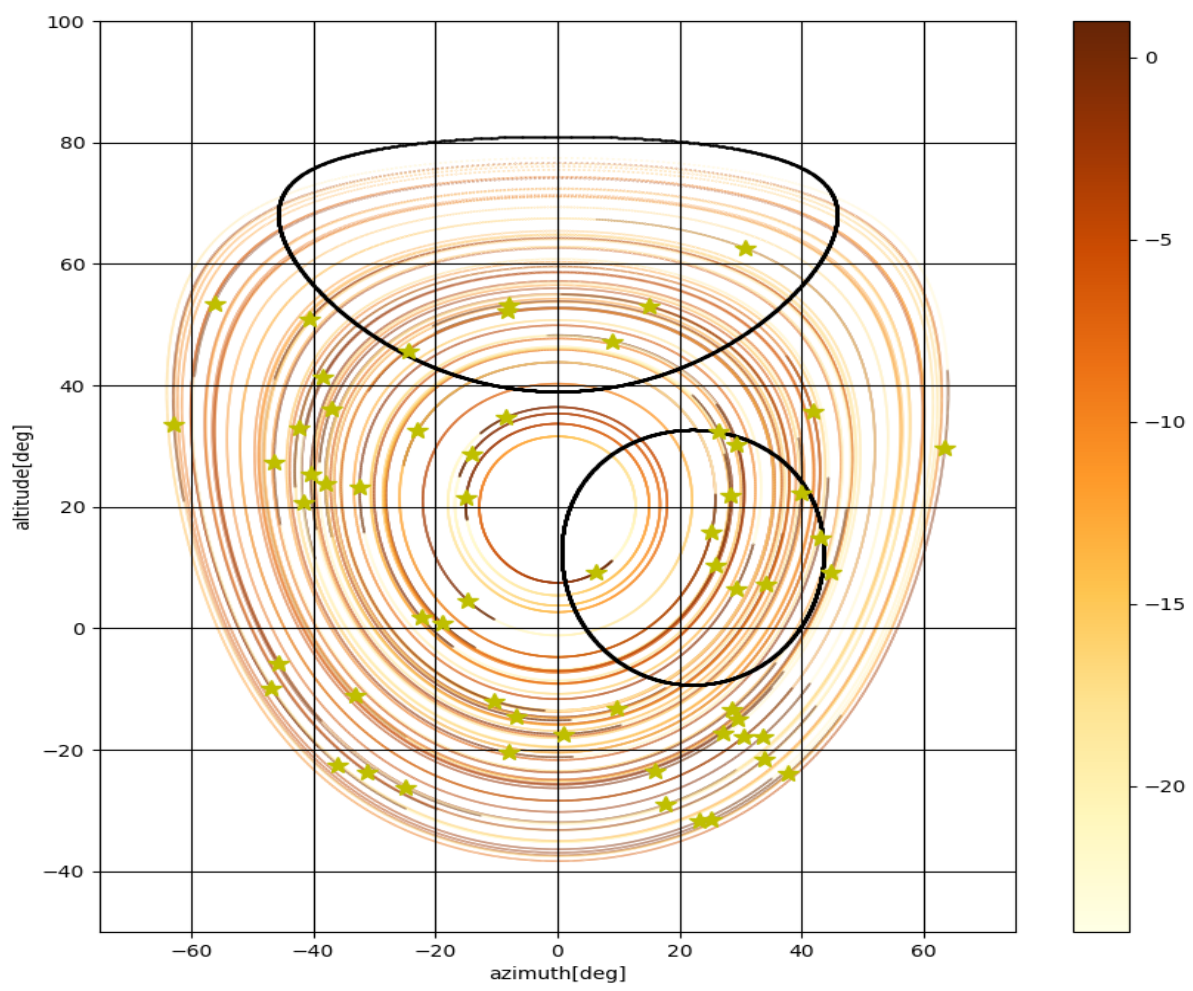

Figure 2: The GRB trajectories in the horizontal coordinate of each our light collector. These trajectories are displayed in the field of view of the light collector, viewing $42^{\circ}$ circle region of which center are located at $($ Alt, Az $)=\left(11.7^{\circ}, 22.1^{\circ}\right)$ and $($ Alt, Az $)=\left(60.0^{\circ}, 0.0^{\circ}\right)$. The black circles represent the FOV of each light collector. The color represent a time from the $\mathrm{t}_{0}$ and time range is $t_{0}-24$ hours to $t_{0}+1$ hour. Star marks represent the GRB place at $t_{0}$.

\section{Acknowledgment}

We thank Satoru Ogawa, Makoto Sasaki and Toshifumi Aoki for valuable advice on this analysis. This work was supported by Toho University and Institute for Cosmic Ray Research, University of Tokyo.

\section{References}

[1] Mészáros, P. 2006, Rep. Prog. Phys., 69, 2259

[2] Rees, M. J., Mészáros, P. 1994, ApJ, 430, L93

[3] Mészáros , P, Rees, M.J., 1997, ApJ, 476, 232

[4] Kumar, P., Panaitescu, A. 2000, ApJ, 541, L51 
[5] Zhang, B., et al. 2006, ApJ, 642, 354

[6] Yamazaki, R., et al. 2006, MNRAS, 369, 311

[7] Zhang B. B., et al. 2009, ApJ, 690, L10

[8] Sari, R., et al. 1998, ApJ, 497, L17

[9] Yamazaki, R., 2009, ApJ, 690, L118

[10] Y.Asaoka, M.Sasaki, Nucl. Instr. A, 647 (2011) 34-38.

[11] Y.Asaoka, Y.Aita, T.Aoki, and M.Sasaki, IEEE Trans. Nucl. Sci. 52（2005） 1774.

[12] Y.Asaoka et al., ApJ, 736 (2011).

[13] Swift GRB Information, https://gcn.gsfc.nasa.gov/swift_grbs.html

[14] Fermi GRB Information, https://gcn.gsfc.nasa.gov/fermi_grbs.html 